Quim. Nova, Vol. 34, No. 4, 710-713, 2011

\title{
VARIACIÓN DEL GRADO DE AVANCE DE REACCIÓN EN SISTEMAS CERRADOS EN EQUILIBRIO QUÍMICO QUE MODIFICAN LA TEMPERATURA A VOLUMEN CONSTANTE
}

\author{
Joan Josep Solaz-Portolés \\ Departament de Didàctica de les Ciències Experimentals i Socials. Universitat de València. Avgda. Tarongers, 4. 46022 València, Spain
}

Recebido em 6/6/10; aceito em 30/10/10; publicado na web em 7/2/11

\begin{abstract}
VARIATION OF EXTENT OF REACTION IN CLOSED CHEMICAL EQUILIBRIUM WHEN CHANGING THE TEMPERATURE AT CONSTANT VOLUME. In this paper it is presented a thermodynamic analysis that aims to find the mathematical expression of the variation of extent of reaction with the infinitesimal variation in the temperature at constant volume of a chemical equilibrium mixture. The goal of this paper is to establish an alternative approach to avoid both the Le Chatelier's principle and the problems that emerge when trying to apply its qualitative statements. This attempt is based on the laws of thermodynamics.
\end{abstract}

Keywords: chemical equilibrium; Le Chatelier's principle; extent of reaction.

\section{INTRODUCCIÓN}

Los libros de texto de Química Física suelen ofrecer la variación del grado de avance de los sistemas cerrados en equilibrio químico con la temperatura, a presión constante, y con la presión, a temperatura constante. Sin embargo, no suelen encontrarse libros de texto en los que se obtenga la variación del grado de avance en sistemas abiertos en equilibrio químico. ${ }^{1}$ Tampoco es fácil encontrar libros de texto que proporcionen la variación del grado de avance de sistemas cerrados en equilibrio químico con la temperatura a volumen constante.

Además, se ha comprobado que los libros de texto de Química que se utilizan es España suelen presentar varias deficiencias en el tema de equilibrio químico. ${ }^{2}$ También se ha puesto en evidencia las confusiones que suelen producirse en los textos en la utilización de las distintas funciones delta, $\Delta G, \Delta_{r} G$ y $\Delta_{r} G^{0 ; 3}$ o la inadecuada presentación, tanto desde del punto de vista didáctico como epistemológico, de las constantes de equilibrio químico (normalmente no se le da apoyo teórico sino empírico). ${ }^{4}$

Por otra parte, el análisis del desplazamiento del equilibrio químico por la modificación de alguna de las variables que lo definen se lleva a cabo en muchos casos en los libros de texto de Química General sobre la base del principio de Le Chatelier como regla cualitativa. ${ }^{5}$ No obstante, las formulaciones cualitativas del principio adolecen de graves deficiencias ${ }^{6-8}$ y pecan de vaguedad porque no se concretan las condiciones de validez, ${ }^{9}$ lo que puede conducir a una amplia gama de errores conceptuales. ${ }^{10}$ Justamente, en la variación de la temperatura de un sistema cerrado a volumen constante de gases en equilibrio químico pueden presentar problemas las formulaciones cualitativas del principio de Le Chatelier, ya que existe la posibilidad de errar en el desplazamiento del equilibrio porque se alteran simultáneamente presión y temperatura. ${ }^{11}$

En este trabajo mostraremos que, tomando como base conceptual los potenciales termodinámicos y la afinidad química, podemos prever el sentido de la evolución de los sistemas cerrados en equilibrio químico que han sido perturbados modificando la temperatura a volumen constante. El planteamiento termodinámico presentado nos posibilitará la obtención de la expresión matemática de la variación del grado de avance con la variación infinitesimal de la temperatura.

\section{POTENCIALES TERMODINÁMICOS Y AFINIDAD QUÍMICA}

Partiendo de las expresiones diferenciales de los potenciales termodinámicos Energía Interna $(U)$, Entalpía $(H)$, Energía Libre de Helmholtz (la representaremos mediante $F$, aunque usualmente se utiliza $A$, para no confundirla con la Afinidad química) y Energía Libre de Gibbs $(G)^{12}$

$$
\begin{gathered}
d U=T d S-P d V+\sum_{i} v_{i} \mu_{i} d \xi \\
d H=T d S+V d P+\sum_{i} v_{i} \mu_{i} d \xi \\
d F=-S d T-P d V+\sum_{i} v_{i} \mu_{i} d \xi \\
d G=-S d T+V d P+\sum_{i} v_{i} \mu_{i} d \xi
\end{gathered}
$$

donde $T$ es la Temperatura, $S$ la Entropía, P la Presión, $V$ el Volumen, $\Sigma v_{i} \mu_{i}$ el sumatorio de los productos de los coeficientes estequiométricos y los potenciales químicos de las especies químicas $i$ que intervienen en la reacción química, y $d \xi$ la variación elemental del grado de avance de una reacción química.

En la última de estas diferenciales totales el coeficiente de $d \xi$, es decir, el sumatorio $\sum_{i} v_{i} \mu_{i}$ representa la velocidad de cambio del potencial energía libre de Gibbs con el grado de avance de la reacción, manteniendo constantes $T$ y $P$. A este coeficiente se le denomina energía libre de Gibbs de reacción $\left(\Delta_{\mathrm{r}} \mathrm{G}\right)$ o, cambiado de signo, afinidad química de la reacción $(A)^{3}$

$$
A=-\Delta_{r} G=-\left(\frac{\partial G}{\partial \xi}\right)_{T, P}=-\sum_{i} v_{i} \mu_{i}
$$

Por otra parte, como la energía libre de Gibbs es una función de estado dependiente de la temperatura, la presión y el grado de avance de la reacción, la afinidad química también será una función de estado cuyas variables naturales serán las mismas. Así pues, $A=A(T, P, \xi)$.

Para los restantes potenciales termodinámicos tendremos que 


$$
\begin{aligned}
& A=-\left(\frac{\partial U}{\partial \xi}\right)_{S, V}=-\sum_{i} v_{i} \mu_{i} \\
& A=-\left(\frac{\partial H}{\partial \xi}\right)_{S, P}=-\sum_{i} v_{i} \mu_{i} \\
& A=-\left(\frac{\partial F}{\partial \xi}\right)_{T, V}=-\sum_{i} v_{i} \mu_{i}
\end{aligned}
$$

Luego de un modo genérico la afinidad química $A$ representa la variación del potencial termodinámico que gobierna el comportamiento del sistema con el grado de avance de la reacción, manteniendo constantes las restantes variables naturales.

Finalmente, las diferenciales de los potenciales termodinámicos pueden quedar expresadas del siguiente modo

$$
\begin{gathered}
d U=T d S-P d V-A d \xi \\
d H=T d S+V d P-A d \xi \\
d F=-S d T-P d V-A d \xi \\
d G=-S d T+V d P-A d \xi
\end{gathered}
$$

\section{CONDICIONES DE EVOLUCIÓN Y DE EQUILIBRIO QUÍMICO}

Teniendo presente que las diferenciales de los distintos potenciales termodinámicos en los procesos irreversibles efectuados manteniendo constantes sus respectivas parejas de variables naturales $(S, V),(S, P),(\mathrm{T}, \mathrm{V})$ y $(T, P)$ deben ser negativas, ${ }^{12}$ la condición de evolución espontánea para una reacción química en esas condiciones puede expresarse como

$$
A d \xi>0
$$

o también como

$$
\Delta_{r} G d \xi<0
$$

Así, el sentido de la evolución de una reacción química, determinado por el signo de $d \xi$, viene dado por $A$ o $\Delta_{r} G$. Un sistema cuya afinidad química es positiva evolucionará en el sentido correspondiente a un aumento en el grado de avance de la reacción $(d \xi>0)$, es decir, de reactivos a productos. Por el contrario, si $A<0$, entonces $d \xi<0$, y el sentido de la evolución será de productos a reactivos.

Cuando un sistema deja de evolucionar macroscópicamente, esto es, ha alcanzado el equilibrio, los potenciales termodinámicos se hacen mínimos y sus diferenciales son iguales a cero. Esto implica que la afinidad química se hace cero. En consecuencia, la condición de equilibrio de un sistema asiento de una reacción química puede escribirse como

$$
A=0
$$

o bien,

$$
\Delta_{r} G=0
$$

\section{Evolución de un sistema en equilibrio químico tras una perturbación}

Supongamos un sistema definido por la variables independientes
$T, P$ y $\xi$ y que se encuentra inicialmente en un estado 1 de equilibrio, por tanto la afinidad química del sistema

$$
A_{1}(T, P, \xi)=0
$$

Si el sistema es perturbado a un estado 2 fuera del equilibrio y que difiere en cantidades infinitesimales $d T, d P$ y $d \xi$ del estado 1 , entonces la afinidad de este nuevo estado será

$$
A_{2}=A_{1}+d A_{1 \rightarrow 2}
$$

en donde teniendo presente que $A_{1}=0$ y que de acuerdo con la Ecuación $13 A_{2} d \xi>0$, se concluye que el sentido de evolución espontánea de una reacción en equilibrio sometida a una perturbación se ajustará a

$$
d A_{1 \rightarrow 2} d \xi>0
$$

Por tanto, si $d A_{1 \rightarrow 2}>0$ implica que $d \xi>0$, es decir, la reacción se desplaza hacia la formación de productos. En cambio, cuando $d A_{1 \rightarrow 2}<0$ se tiene que $d \xi<0$, por lo que la reacción se desplaza de productos a reactivos.

Nuestro sistema, tras la correspondiente evolución, que algún autor prefiere denominarla relajación, ${ }^{13}$ alcanzará un nuevo estado de equilibrio, al que llamamos estado 3 . Hemos de señalar que aunque los estados 1 y 3 son de equilibrio diferirán en los valores de $T, P$ y $\xi$. Para el estado 3 tendremos que

$$
A_{3}\left(T^{\prime}, P^{\prime}, \xi^{\prime}\right)=0
$$

Como es obvio, la variación de la afinidad química al pasar el sistema del estado 1 al 3 tras la perturbación debe ser cero, $d A_{1 \rightarrow 3}=0$.

Todo lo dicho hasta aquí en este apartado puede resumirse en el siguiente diagrama (Figura 1).

Del anterior diagrama se desprende que en el estudio de la perturbación del equilibrio químico se pueden llevar a cabo dos análisis. El primero de ellos examina el paso de un estado de equilibrio (estado 1) a un estado perturbado (2), y el sentido de la evolución del sistema viene dado por el signo de $d A_{1 \rightarrow 2}$. En el segundo, se estudia el tránsito entre el estado inicial de equilibrio (estado 1) y el estado de nuevo equilibrio (estado 3 ) alcanzado tras la perturbación, que tiene lugar sin que varíe la afinidad química $d A_{1 \rightarrow 3}=0$.

Por ello, en el estudio de los efectos producidos por una perturbación sobre un sistema en equilibrio químico podemos, por ejemplo, partir de la expresión

$$
d A=\left(\frac{\partial A}{\partial T}\right)_{P, \xi} d T+\left(\frac{\partial A}{\partial P}\right)_{T, \xi} d P+\left(\frac{\partial A}{\partial \xi}\right)_{T, P} d \xi
$$

y si llevamos a cabo el primer análisis (paso del estado 1 de equilibrio al estado 2 perturbado), entonces el signo de $d A$ nos indicará el sentido de la evolución del sistema. Por el contrario, si realizamos el segundo análisis (paso del estado 1 de equilibrio al estado 3, también de equilibrio), entonces $d A=0$, y de ahí extraeremos las oportunas conclusiones a través de la variación del grado de avance con la variable que ha sido modificada en la perturbación.

\section{Modificación infinitesimal de la temperatura a volumen constante en sistemas cerrados que no alteran su composición. Análisis desde el equilibrio inicial al equilibrio final}

Hemos visto en un parágrafo anterior que de un modo genérico la afinidad química representa la variación del potencial termodinámico que gobierna el comportamiento del sistema con el grado de avance de 
Estado 1

Sistema en

equilibrio

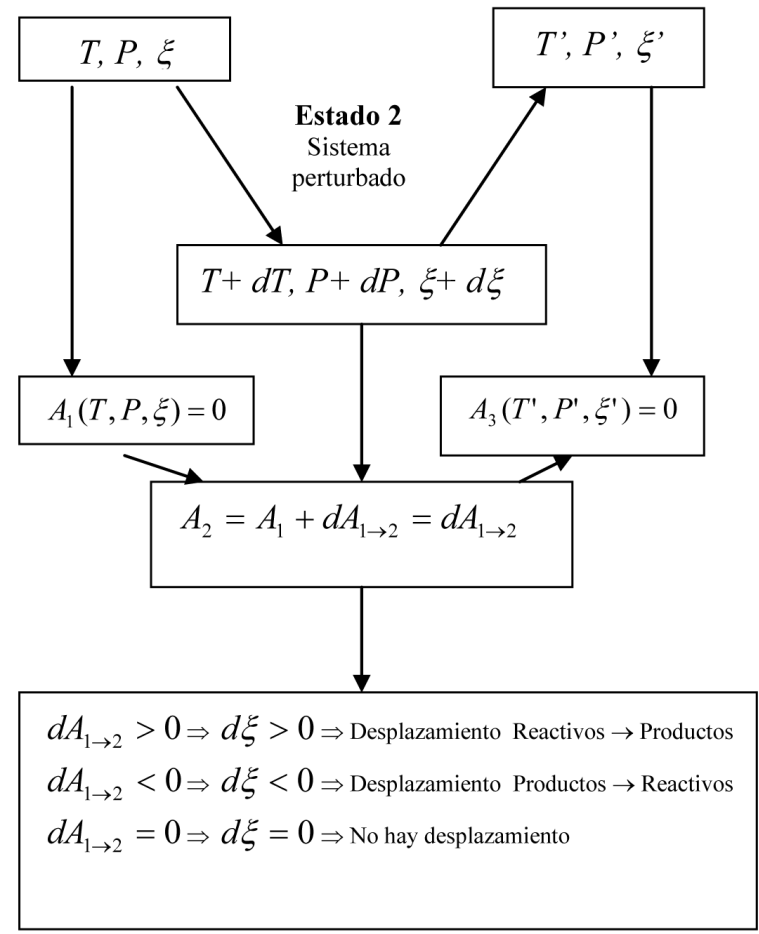

Figura 1. Diagrama que muestra los estados, los valores de la afinidad química y de sus variaciones infinitesimales, así como el sentido del desplazamiento de un sistema en equilibrio químico sometido a una perturbación

la reacción, manteniendo constantes las restantes variables naturales. En concreto, si la temperatura y el volumen son constantes

$$
A=-\left(\frac{\partial F}{\partial \xi}\right)_{T, V}=-\sum_{i} v_{i} \mu_{i}
$$

Así que, como las variables naturales del sistema son $T, V$ y $\xi$, la diferencial total de $A$ cambiada de signo será posible expresarla

$d\left(\frac{\partial F}{\partial \xi}\right)_{T, V}=\left[\frac{\partial}{\partial T}\left(\frac{\partial F}{\partial \xi}\right)_{T, V}\right]_{V, \xi} d T+\left[\frac{\partial}{\partial V}\left(\frac{\partial F}{\partial \xi}\right)_{T, V}\right]_{T, \xi} d V+\left(\frac{\partial^{2} F}{\partial \xi^{2}}\right)_{T, V} d \xi$

Por otro lado, teniendo presente las diferencial del potencial termodinámico energía libre de Helmholtz

$$
d F=-S d T-P d V-\mathrm{A} d \xi
$$

y la igualdad de las derivadas parciales segundas cruzadas, se coligen las siguientes expresiones

$$
\begin{aligned}
& {\left[\frac{\partial}{\partial T}\left(\frac{\partial F}{\partial \xi}\right)_{T, V}\right]_{V, \xi}=\left[\frac{\partial}{\partial \xi}\left(\frac{\partial F}{\partial T}\right)_{V, \xi}\right]_{T, V}=-\left(\frac{\partial S}{\partial \xi}\right)_{T, V}} \\
& {\left[\frac{\partial}{\partial V}\left(\frac{\partial F}{\partial \xi}\right)_{T, V}\right]_{T, \xi}=\left[\frac{\partial}{\partial \xi}\left(\frac{\partial F}{\partial V}\right)_{T, \xi}\right]_{T, V}=-\left(\frac{\partial P}{\partial \xi}\right)_{T, V}}
\end{aligned}
$$

Con lo cual, efectuando substituciones pertinentes, la expresión diferencial (23) es susceptible de ser formulada del modo siguiente

$$
d\left(\frac{\partial F}{\partial \xi}\right)_{T, V}=-\left(\frac{\partial S}{\partial \xi}\right)_{T, V} d T-\left(\frac{\partial P}{\partial \xi}\right)_{T, V} d V+\left(\frac{\partial^{2} F}{\partial \xi^{2}}\right)_{T, V} d \xi
$$

o también como

$$
d A=\left(\frac{\partial S}{\partial \xi}\right)_{T, V} d T+\left(\frac{\partial P}{\partial \xi}\right)_{T, V} d V-\left(\frac{\partial^{2} F}{\partial \xi^{2}}\right)_{T, V} d \xi
$$

que en un cambio de temperatura a volumen constante y considerando un tránsito entre dos estados de equilibrio, quedará

$$
d A=\left(\frac{\partial S}{\partial \xi}\right)_{T, V} d T-\left(\frac{\partial^{2} F}{\partial \xi^{2}}\right)_{T, V} d \xi=0
$$

Por otra parte, si partimos de la definición del potencial termodinámico energía libre de Helmholtz $F=U-T S$, y la derivamos con respecto al grado de avance a $T$ y $V$ constantes

$$
\left(\frac{\partial F}{\partial \xi}\right)_{T, V}=\left(\frac{\partial U}{\partial \xi}\right)_{T, V}-T\left(\frac{\partial S}{\partial \xi}\right)_{T, V}
$$

En el equilibrio

$$
\left(\frac{\partial F}{\partial \xi}\right)_{T, V}=0
$$

por tanto, tendremos que

$$
\left(\frac{\partial S}{\partial \xi}\right)_{T, V}=\frac{1}{T}\left(\frac{\partial U}{\partial \xi}\right)_{T, V}
$$

y como para gases ideales ${ }^{14}$

$$
\left(\frac{\partial U}{\partial \xi}\right)_{T, V} \approx\left(\frac{\partial U}{\partial \xi}\right)_{T, P} \approx \Delta_{r} U^{0}
$$

podemos finalmente deducir de la expresión diferencial (29) que

$$
\frac{d \xi}{d T}=\frac{\Delta_{r} U^{0}}{T F^{\prime \prime}}
$$

en donde $F^{\prime \prime}=\left(\frac{\partial^{2} F}{\partial \xi^{2}}\right)_{T, V}$ que, si el equilibrio es considerado estable, es positiva (condición de mínimo del potencial termodinámico energía libre de Helmoltz).

Dos hechos deben ser destacados de esta última ecuación. En primer lugar, nos indica que el efecto del cambio simultáneo de dos variables intensivas, temperatura y presión (téngase en cuenta que la modificación de temperatura a volumen constante en un sistema en el que participan gases provoca una alteración de la presión del mismo), puede ser predicho solamente mediante el signo de la variación de la temperatura. En segundo lugar, el sentido del desplazamiento de la mezcla en equilibrio viene regido por el signo de la energía interna de reacción estándar $\Delta_{r} U^{0}$, lo que pone de manifiesto la incorrección del uso indiscriminado de $\Delta H^{0}$ para este mismo fin.

\section{CONCLUSIONES}

La utilización de la Leyes de la Termodinámica nos ha conducido a la posibilidad de llevar a cabo dos análisis que permiten abordar con precisión y rigor el estudio de sistemas en equilibrio químico 
tras la perturbación de los mismos. Escoger uno u otro, o una forma simplificada de ambos, estará en función de la situación didáctica en la que nos encontremos. En el caso que hemos estudiado, modificación de la temperatura de un sistema de gases en equilibrio químico a volumen constante, la aplicación de uno de estos análisis -del sistema en equilibrio inicial al equilibrio final- nos ha permitido obtener la expresión matemática de la variación del grado de avance de reacción con la variación infinitesimal de la temperatura. El otro análisis -del sistema en equilibrio inicial al sistema perturbado- puede verse desarrollado en el trabajo de Solaz-Portolés. ${ }^{15}$

En cualquier caso, estos análisis permiten soslayar la utilización indiscriminada del principio de Le Chatelier como regla meramente cualitativa, que tantos problemas puede generar. No ocultaremos, sin embargo, que el tratamiento propuesto presenta cierto grado de complejidad frente a la metafísica simplicidad del enunciado del principio de Le Chatelier que usualmente se presenta en los libros de texto. En relación con esto, diremos que se puede seguir utilizando dicho principio teoretizándolo, ${ }^{16}$ es decir, insertándolo en un cuerpo de teoría - La Termodinámica-. De esta forma, el principio deja de ser una hipótesis empírica aislada para convertirse en un teorema derivado de las Leyes fundamentales de la Termodinámica, con las consiguientes ventajas didácticas. ${ }^{9}$

\section{REFERENCIAS}

1. Solaz-Portolés, J. J.; Quílez, J.; Chem. Educ. Res. Pract. Eur. 2001, 2, 303.

2. Solaz-Portolés, J. J.; Rev. Chil. Educ. Cient. 2007, 6, 13.

3. Solaz-Portolés, J. J.; Quílez, J.; Educ. Quím. 2001, 12, 103.

4. Solaz-Portolés, J. J.; Quím. Bras., in press.

5. Quílez, J.; Solaz-Portolés, J. J.; Castelló, M.; Sanjosé, V.; Enseñanza de las Ciencias 1993, 11, 282.

6. Quílez, J.; Solaz-Portolés, J. J.; Afinidad 1994, 454, 435

7. Solaz-Portolés, J. J.; Quílez, J.; Rev. Mex. Fís. 1995, 41, 128.

8. Quílez, J.; Solaz-Portolés, J. J.; Educ. Quím. 1996, 7, 202.

9. Quílez, J.; Solaz-Portolés, J. J.; Cad. Cat. Ens. Fís. 1995, 12, 123.

10. Quílez, J.; Solaz-Portolés, J. J.; J. Res. Sci. Teach. 1995, 33, 939.

11. Bridgart, G.; Kemp, H.; Austral. Sci. Teach. J. 1985, 31, 60.

12. Sanfeld, A. In Physical Chemistry; Jost, W., ed.; Academic Press: New York, 1971, vol. 1, chap. 2A.

13. Schuffenecker, L. ; Proust, B. ; Scacchi, G. ; Foucaut, J. F. ; Martel, L. ; Bouchy, M. ; Thermodynamique Chimique et Cinetique Chimique, $1^{\text {st }}$ ed., Tecnique et Documentation: Paris, 1991.

14. Brenon-Audat, F. ; Busquet, C. ; Mesnil, C. ; Thermodynamique Chimique, $1^{\text {st }}$ ed., Hachette: Paris, 1993.

15. Solaz-Portolés, J. J.; Termodinámica y equilibrio químico, $1^{\text {st }}$ ed., C. F. Tomás y Valiente UNED: Valencia, 2002.

16. Bunge, M.; La investigación científica, $2^{\text {nd }}$ ed., Editorial Ariel: Barcelona, 1985. 